\title{
ROR2 regulates the survival of murine osteosarcoma cells in lung capillaries
}

\author{
Diem Thi Phuong Tran'1, Takahiro Kuchimaru², Mongkol Pongsuchart' ${ }^{1}$, Kha The Nguyen ${ }^{1}$, John Clyde Co \\ Soriano', Tetsuya Kadonosono ${ }^{1}$, Shinae Kizaka-Kondoh ${ }^{1}$
}

'School of Life Science and Technology, Tokyo Institute of Technology, Yokohama 226-8501, Japan.

${ }^{2}$ Center for Molecular Medicine, Jichi Medical University, Tochigi 329-0498, Japan.

Correspondence to: Dr. Shinae Kizaka-Kondoh, School of Life Science and Technology, Tokyo Institute of Technology, $4259-B 60$ Nagatsuta-cho, Midori-ku, Yokohama 226-8501, Japan. E-mail: skondoh@bio.titech.ac.jp

How to cite this article: Tran DTP, Kuchimaru T, Pongsuchart M, Nguyen KT, Co Soriano JC, Kadonosono T, Kizaka-Kondoh S. ROR2 regulates the survival of murine osteosarcoma cells in lung capillaries. J Cancer Metastasis Treat 2020;6:47.

http://dx.doi.org/10.20517/2394-4722.2020.104

Received: 22 Sep 2020 First Decision: 10 Nov 2020 Revised: 10 Nov 2020 Accepted: 24 Nov 2020 Published: 10 Dec 2020

Academic Editor: lan Judson Copy Editor: Cai-Hong Wang Production Editor: Jing Yu

\begin{abstract}
Aim: Lung metastasis is a leading cause of death in patients with osteosarcoma (OS). No effective therapy exists that improves the five-year overall survival rate of OS patients with metastasis. Therefore, finding novel therapeutic targets will help develop new treatment strategies for OS patients with lung metastasis.
\end{abstract}

Methods: Based on analysis of gene expression profiles between sublines of the Dunn OS LM8 cell line with high (LM8-H) and low (LM8-L) metastatic ability, we have identified Wnt signal-related genes that play an important role in lung metastasis of OS. Function of the genes was investigated by establishing sublines of gene knockout and assessing their metastatic ability using a mouse lung metastasis model. The molecular mechanism underlying the function of the genes was further investigated by in vitro experiments.

Results: We have identified that receptor tyrosine kinase-like orphan receptor 2 (ROR2), a receptor of the noncanonical Wnt signaling pathway, was involved in OS cell survival in lung capillaries during metastasis. LM8-H knocked out of Ror2 (H/Ror2-KO) significantly reduced lung metastasis by decreasing the viability in lung capillaries $48 \mathrm{~h}$ after intravenous injection. In vitro study revealed that ROR2 increased anoikis resistance through AKT activation. Reconstitution of ROR2 expression in $\mathrm{H} /$ Ror2-KO cells restored their metastatic ability and viability in lung capillaries. 
Conclusion: The results demonstrate a novel ROR2 function in OS lung metastasis and may inform new treatment strategies for OS patients.

Keywords: Osteosarcoma, lung metastasis, cell survival, lung capillary, ROR2

\section{INTRODUCTION}

Osteosarcoma (OS) is a primary malignant bone tumor that typically occurs in the long bones, with peak incidence in adolescents and young adults ${ }^{[1]}$. Distant metastases of OS, such as lung metastases, are hard to control and are usually associated with poor prognosis. Recent treatment advances involving combinations of chemotherapy, surgery, and other clinical applications have increased the survival rate of OS patients without lung metastasis to $60 \%$. However, the survival rate of OS patients with lung metastasis has not improved over the past 30 years $^{[2]}$. A better understanding of the molecular mechanism behind the OS metastatic process will facilitate the development of new therapeutic strategies for improving the outcomes of patients with OS.

Wnt signaling plays essential roles in the malignant process of OS. The Wnt signaling transduction cascades are classified into two pathways: canonical ( $\beta$-catenin-dependent) and noncanonical ( $\beta$-cateninindependent) pathways ${ }^{[3]}$. The factors of canonical Wnt signaling, such as Wnt3a, Wnt10, Lef1, and $\beta$-catenin, are involved in the development and malignant progression of $\mathrm{OS}^{[4]}$. In contrast, noncanonical Wnt signaling is known to be transduced by binding Wnt5a to Frizzled or receptor tyrosine kinase-like orphan receptors (RORs), and promotes OS malignancies through phosphatidylinositol-3 kinase (PI3K)/ AKT signaling $^{[5]}$ and c-Jun N-terminal kinase (JNK) pathway ${ }^{[6]}$.

Receptor tyrosine kinase-like orphan receptor 2 (ROR2) is characterized by three main domains: the intracellular tyrosine kinase domain, extracellular Frizzled-like cysteine-rich domain, and membraneproximal Kringle domain ${ }^{[7-9]}$. The extracellular domain of ROR2 is assumed to mediate protein-protein interactions, and ROR2 functions as an alternative or coreceptor for Wnt5a, a representative noncanonical Wnt ligand ${ }^{[10]}$. ROR2 activates JNK pathway ${ }^{[11,12]}$ and inhibits the $\beta$-catenin-dependent Wnt pathway ${ }^{[10-12]}$. ROR2 is overexpressed in $73.8 \%$ of OS samples and is correlated with tumor metastasis ${ }^{[13]}$, suggesting a pivotal role in OS progression. However, the molecular mechanism of ROR2 function in OS lung metastasis has not been completely elucidated.

The process of OS metastasis to the lungs includes migration, intravasation into the circulation, survival in the circulation and lung capillaries, and extravasation into the lungs. In the lung capillaries, most of OS cells die for various reasons such as mechanical stress and lack of attachment to proper extracellular matrix (ECM). Anoikis is a type of apoptosis induced by the lack of proper ECM attachment necessary for cell maintenance in tissues ${ }^{[14]}$. Tumor cells gain the ability to resist anoikis and undergo the metastatic process, prolonging their survival time and promoting migration and colonization at secondary sites ${ }^{[15]}$. Several mechanisms of anoikis resistance in cells of epithelial origin have been described ${ }^{[14]}$. However, there are few studies on anoikis resistance in cells of non-epithelial origin, such as OS cells.

We have recently established LM8 murine OS sublines with different abilities to metastasize to the lung using an in vivo image-guided screening system ${ }^{[16]}$. Based on the microarray data of the LM8 sublines, we recently identified a novel lymphoid enhancer-binding factor 1 (LEF1)-cytoglobin (CYGB) axis as a part of noncanonical Wnt signaling pathway that promotes OS cell extravasation into the lungs ${ }^{[16]}$. In the present study, we aimed to search for new therapeutic targets using the same techniques to develop treatment strategies for OS lung metastases. 


\section{METHODS}

\section{Gene-set enrichment analysis}

Differentially expressed genes were selected from the microarray data of LM8 sublines and subjected to gene set enrichment analysis of the Kyoto Encyclopedia of Genes and Genomes. The analysis was performed with the clusterProfile package using R/Bioconductor. The reference gene set used was the Wnt signaling pathway (mm0431) set.

\section{Cell culture}

The LM8 cell subline was provided by Dr. Hideki Yoshikawa (Osaka University). LM8 cell line with high (LM8-H) and low (LM8-L) was established from LM8 in a previous study through the in vivo imageguided screening system ${ }^{[16]}$. Murine vascular endothelium cells, bEnd3, were obtained from the American Type Culture Collection. All cells used in this study were cultured in $5 \%$ fetal bovine serum (FBS) Dulbeccos Modified Eagle's Medium (DMEM) supplemented with penicillin (100 U/mL) and streptomycin $(100 \mu \mathrm{g} / \mathrm{mL})$.

\section{Reverse transcription PCR and quantitative PCR}

Total RNA was extracted using the RNeasy ${ }^{\bullet}$ Mini Kit (Qiagen, Hilden, Germany) according to the manufacturer's protocol. One microgram of total RNA was reverse-transcribed using the Oligo(dT)20 primer (Toyobo, Osaka, Japan) and ReverTra Ace (Toyobo). Quantitative PCR (qPCR) and reverse transcription PCR (RT-PCR) were carried out using the Thunderbird ${ }^{\star}$ SYBR qPCR Mix (Toyobo) and EmeraldAmp ${ }^{\circ}$ GT PCR Master Mix (Takara Bio, Shiga Japan), respectively.

\section{Western blotting}

Cells were lysed in RIPA buffer (50 mmol/L Tris $\mathrm{HCl}, \mathrm{pH}$ 8.0; $150 \mathrm{mmol} / \mathrm{L} \mathrm{NaCl}, 1 \% \mathrm{NP}-40,0.5 \%$ sodium deoxycholate, $0.1 \%$ SDS) containing a protease cocktail inhibitor (Nacalai Tesque, Kyoto, Japan), and protein concentration was determined using the Pierce BCA protein assay kit (Thermo Fisher Scientific, Waltham, MA). Proteins were separated by electrophoresis on a $10 \%$ acrylamide gel, transferred to a hydrophilic polyvinylidene fluoride membrane (Merck, Kenilworth, NJ) and blocked with 5\% skim milk or $5 \%$ bovine serum albumin in TBST ( $20 \mathrm{mmol} / \mathrm{L}$ Tris, $\mathrm{pH} 7.5 ; 150 \mathrm{mmol} / \mathrm{L} \mathrm{NaCl}, 0.1 \%$ Tween 20 ). The membrane was then probed with relevant primary antibodies [anti-AKT (\#2920S, 1:5000), anti-phosphoAKT (Ser473; \#4060s, 1:5000), anti-ROR2 (\#88639s, 1:5000), and anti-GAPDH (\#2118s; Cell Signaling Technology, 1:5000)], and secondary antibodies [anti-mouse IgG HRP-linked antibody (\#7076, 1:5000) and anti-rabbit IgG HRP-linked antibody (\#7074, 1:5000, Cell Signaling Technology)]. The resultant membranes were washed with TBST and detected by ImageQuant LAS 4000 (GE Healthcare Life Science, Marlborough, MA) after processing with Chemi-Lumi One (Nacalai Tesque). To investigate the effect of AKT inhibition, cells were treated with $50 \mathrm{nmol} / \mathrm{L}$ MK2206 in 5\% FBS DMEM for $24 \mathrm{~h}$.

\section{Gene knockout using the CRISPR-Cas9 system}

Ror 2 gene knockout (KO) was performed in LM8-H cells to establish the cell line H/Ror2-KO by using the CRISPR-Cas9 system. The sequence of the guide RNA (5'-caccgTCGTGGCTCTTGCACAACCG-3') was used for targeting Ror2. The Ror2 guide RNA was inserted into a unique BbsI site of the pX330 plasmid (42230; Addgene). The cells whose genomes were correctly edited by the CRISPR-Cas9 system were selected by using a fluorescence indicator system with the pCAG/EGxxFP plasmid ${ }^{[17]}$, provided by Dr. Masahito Ikawa (Osaka University). GFP-positive cells were picked up, and ROR2 protein expression level was validated by western blotting.

\section{Establishment of cell lines with ROR2-overexpression}

The cDNA of Ror2 (NM_013846.4) was amplified using the KOD ${ }^{\circ}$ FX Kit (Toyobo) using the following primer set: 5'-TGGAATTCTGCAGATATGGCTCGGGGCTGGGTG-3' and 5'-GCCACTGTGCTGGA TTCAGGCTTCAAGCTGGACATG-3'. The Ror 2 cDNA fragment was cloned into the pcDNA3.1-myc- 
His vector (Invitrogen, Carlsbad, CA) at the EcoRV site using the Infusion Cloning Enhancer kit (Takara Bio). LM8-L and H/Ror2-KO cells were transfected with the plasmid by the NEPA21 type II electroporator (NEPAGENE, Chiba, Japan), and appropriate transfectants were cloned to establish L/ROR2 and KO/ROR2 after culturing with $\mathrm{G} 418(2 \mathrm{mg} / \mathrm{mL})$ selection medium.

\section{Proliferation assay}

Cell proliferation was evaluated with the water-soluble tetrazolium salt 1 (WST-1) reagent (Sigma Aldrich, St. Louis, MO) according to the manufacturer's instructions. Cells $\left(1 \times 10^{3}\right.$ cells $/ 100 \mu \mathrm{L}$ culture medium $)$ were seeded in 96 -well plates. After culturing for 24,48 , or $72 \mathrm{~h}$, the medium was removed and $100 \mu \mathrm{L}$ of WST-1-containing medium (10-fold dilution) was added to each well. The cells were further incubated for $3 \mathrm{~h}$, and then the absorbance of each well was measured at $450 \mathrm{~nm}$ with a reference wavelength of $750 \mathrm{~nm}$ after shaking the plates for 1 min with the microplate reader Model 680XR (Bio-Rad, Hercules CA).

\section{Mice}

Male $\mathrm{C} 3 \mathrm{H}$ mice were obtained from the Charles River Laboratory (Yokohama, Japan). All mice used were 6-8 weeks of age and were housed in the animal facilities at the Tokyo Institute of Technology. Animal experiments were performed with the approval of the Animal Ethics Committees of the Tokyo Institute of Technology (no. D20170004-2) and in accordance with the Ethical Guidelines for Animal Experimentation of the Tokyo Institute of Technology.

\section{Analysis of OS lung metastasis model}

The mice were injected intravenously with $1 \times 10^{6} \mathrm{LM} 8$ cells in $100 \mu \mathrm{L}$ of PBS. Twenty days after inoculation, isolated lungs were embedded in an optimal cutting temperature compound (Sakura Finetechnical Co., Ltd, Tokyo, Japan) and stored at $-80{ }^{\circ} \mathrm{C}$ overnight. Lungs were divided into cryosections of $10 \mu \mathrm{m}$ in thickness and then fixed in $4 \%$ paraformaldehyde. Fixed lung cryosections were then stained with hematoxylin and eosin (HE) and observed under a microscope BZ-X710 (Keyence, Osaka, Japan). A whole lung image was obtained by stitching together partial lung images using BZ-X analyzer software (Keyence).

\section{Analysis of tumor cells in lungs}

Cells were labeled with $100 \mu \mathrm{mol} / \mathrm{L}$ CellTracker ${ }^{\circledR}$ Green (Thermo Fisher Scientific) and intravenously injected into $\mathrm{C} 3 \mathrm{H}$ mice $\left(1 \times 10^{6}\right.$ cells $\left./ 100 \mu \mathrm{L} \mathrm{PBS}\right)$. DyLight ${ }^{\circ}$ 594-labeled isolectin B4 $(6 \mathrm{mg} / \mathrm{kg}$; Vector Laboratories, Burlingame, CA) was injected intravenously to stain endothelial cells 5 min before dissecting the mice. The lungs were collected and observed under confocal microscopy (Carl Zeiss, Oberkochen, Germany). The number of fluorescently labeled cells in the three microscope fields of each lung was quantified using ImageJ software (http://rsb.info.nih.gov/ij//) ${ }^{[18]}$. Results are shown as the average number of cells per field. Each group was analyzed in triplicates. For measuring the fluorescence intensity of the lungs, lung lysate was prepared with RIPA buffer and well-homogenized. The supernatant was then collected, and the fluorescence intensity was measured using Infinite F500 (Tecan, Männedorf, Switzerland) with a filter set for CellTracker ${ }^{\circledR}$ Green $(E x / E m=480 \mathrm{~nm} / 517 \mathrm{~nm})$.

\section{Anoikis assay}

Poly(2-hydroxyethyl methacrylate) (poly-HEMA; Thermo Fisher Scientific) was completely dissolved in $95 \%$ ethanol $(20 \mathrm{mg} / \mathrm{mL})$. Plates were coated with poly-HEMA solution and dried on a clean bench overnight. Cells $\left(5 \times 10^{4}\right)$ in serum-free medium were seeded in a poly-HEMA-coated 24-well plate. After $24 \mathrm{~h}$, calcein AM (Thermo Fisher Scientific) and ethidium homodimer-1 (Thermo Fisher Scientific) were added at final concentrations of $2 \mu \mathrm{mol} / \mathrm{L}$ and $4 \mu \mathrm{mol} / \mathrm{L}$, respectively, and the cells were further incubated for $30 \mathrm{~min}$. The fluorescent signal was observed under fluorescence microscopy and quantified with the BZ-X Analyzer (Keyence). To investigate the effect of AKT on LM8 anoikis, the AKT inhibitor MK2206 
(Nacalai Tesque) was prepared with dimethyl sulfoxide (DMSO), and the cells were cultured for $24 \mathrm{~h}$ in poly-HEMA-treated plates containing $50 \mathrm{nmol} / \mathrm{L}$ MK2206 in 5\% FBS DMEM.

\section{Statistical analysis}

Data were statistically analyzed with a two-sided Student's $t$-test. $P$-values of less than 0.05 were considered statistically significant.

\section{RESULTS}

\section{ROR2 function was required for OS lung metastasis}

Our recent study using the LM8 sublines (LM8-H and LM8-L) and their gene expression microarray data revealed that noncanonical Wnt signaling is associated with LM8 extravasation into lung tissue via the LEF1-CYGB axis ${ }^{[16]}$. The microarray data of LM8-H and LM8-L was used to search for genes associated with the noncanonical Wnt pathway. Gene set enrichment analysis ${ }^{[19]}$ identified several Wnt signalingrelated genes that showed higher expression in LM8-H than in LM8-L [Figure 1A]. Mapping of these genes using Kyoto Encyclopedia of Genes and Genomes ${ }^{[20]}$ and clusterProfile package using R/Bioconductor indicated that ROR $1 / 2$ were receptors for the noncanonical Wnt signaling that is likely involved in the lung metastasis of LM8 [Supplementary Figure 1]. Expression levels of noncanonical Wnt signaling receptor genes Ror1, Ror2, and Frizzled class receptor 1 ( Fzd1) were examined by RT-PCR. Ror2, but not Ror 1 or Fzd1, was expressed at a significantly higher level in LM8-H than in LM8-L [Figure 1B]. Furthermore, the mRNA level of Ror2 was well-correlated with the metastasis-free survival of OS patients [Supplementary Figure 2]. Therefore, we focused on ROR2 for further analysis. The effect of deletion of Ror2 [Figure 1C] on the proliferation and metastatic potential of $\mathrm{LM} 8-\mathrm{H}$ was first investigated. The proliferation rate of $\mathrm{H} /$ Ror2$\mathrm{KO}$ was similar to that of $\mathrm{LM} 8-\mathrm{H}$ and LM8-L [Figure 1D], indicating that ROR2 function is not involved in proliferation. The size and number of foci in the lungs injected with $\mathrm{H} /$ Ror2-KO were significantly smaller than those in the lungs injected with LM8-H [Figure $1 \mathrm{E}$ ]. These data demonstrated the critical role of ROR2 function in LM8 lung metastasis.

\section{ROR2 was necessary for LM8 survival in lung capillaries}

To determine how ROR2 was involved in the extravasation process in vivo, the LM8 sublines in the lungs were examined at $30 \mathrm{~min}$ and $48 \mathrm{~h}$ post-injection because the tumor cells had reached the lungs by $30 \mathrm{~min}$ and began extravasation into lung tissues by $48 \mathrm{~h}$ post-injection. The LM8 sublines were labeled with a green fluorescent dye before injection, and removed lungs were observed under an inverted confocal fluorescence microscope. Similar numbers of fluorescently labeled cells were observed in the lungs injected with $\mathrm{LM} 8-\mathrm{H}$ and $\mathrm{H} /$ Ror2-KO 30 min after injection [Figure 2A], indicating that lack of ROR2 function did not affect the survival of circulating LM8 cells before reaching the lungs. On the other hand, $48 \mathrm{~h}$ after injection, the number of fluorescently labeled cells in lungs injected with $\mathrm{H} /$ Ror2-KO was significantly reduced compared to that in lungs injected with LM8-H [Figure 2A]. The reduction reflects that more H/ Ror2-KO die in lung capillaries than LM8-H, suggesting that ROR2 function was involved in the survival of LM8 cell in lung capillaries prior to extravasation. To confirm ROR2's function, LM8-L, LM8- H, H/Ror2KO, ROR2-overexpressing LM8-L (L/ROR2), and ROR2-overexpressing H/Ror2-KO (KO/ROR2) [Figure 2B] were examined at $48 \mathrm{~h}$ post-injection in terms of their survival in lung capillaries. Significantly higher fluorescence intensity was detected in lung lysates from mice injected with the LM8 sublines expressing high ROR2 (LM8-H, L/ROR2, and KO/ROR2) compared to that in the lung lysates from mice injected with the LM8 sublines expressing low ROR2 (LM8-L and $\mathrm{H} /$ Ror2-KO) [Figure $2 \mathrm{C}$ ]. These results confirmed a correlation between ROR2 expression and LM8 survival in lung capillaries.

\section{The AKT signaling pathway was involved in ROR2-induced anoikis resistance in LM8}

Tumor cells that have reached the lung capillaries are stressed mechanically because the blood vessels are smaller in diameter and are less compliant ${ }^{[21]}$. This mechanical stress causes cell death in up to more 
A

\section{Position in the Ranked List of Genes}

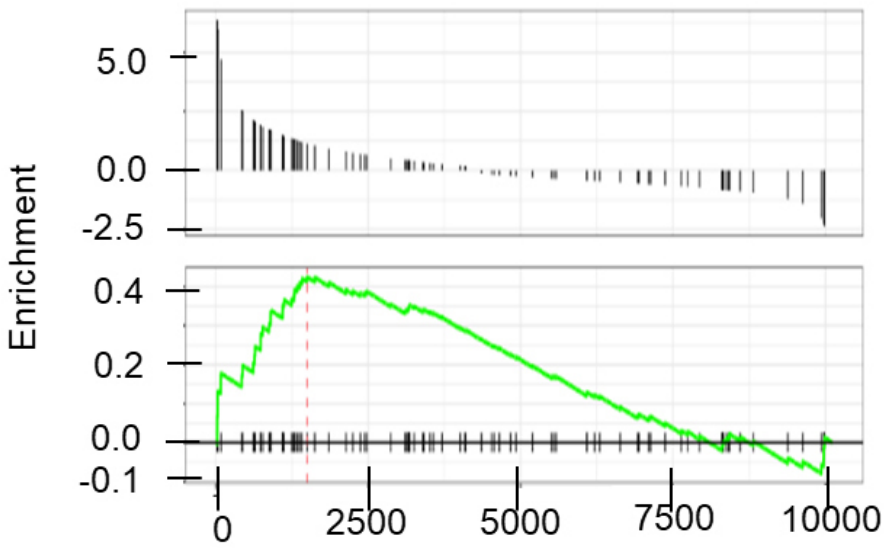

NES $=1.5554410$

$\mathrm{P}=0.011709602$
B

LM8-H LM8-L

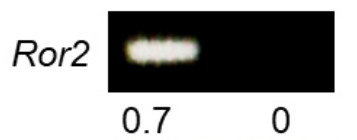

Ror1

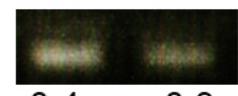

0.4

Fzd1

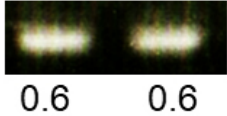

C

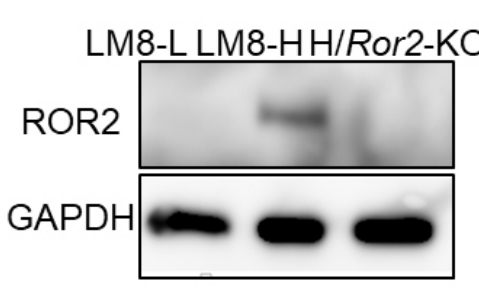

D

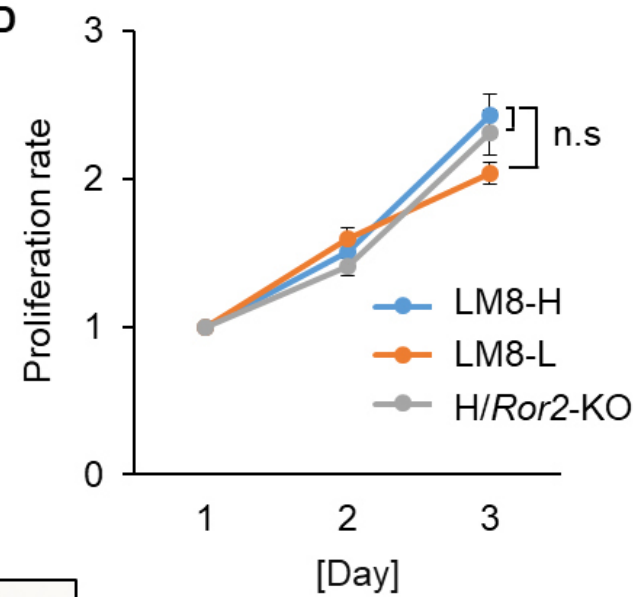

E

$\mathrm{H} /$ Ror2-KO
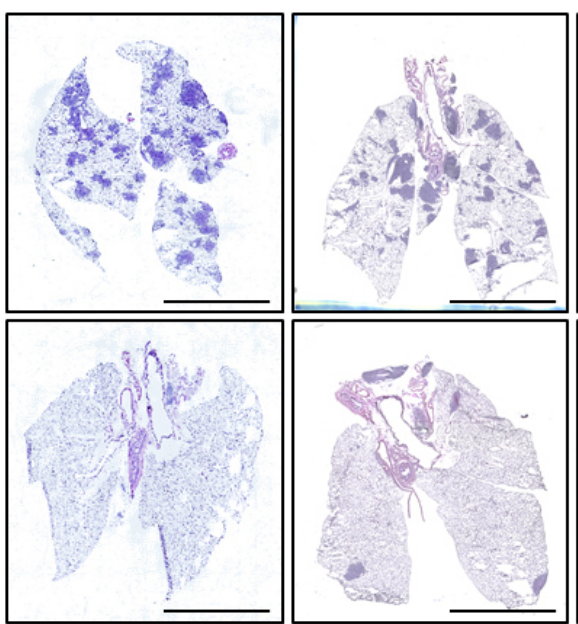

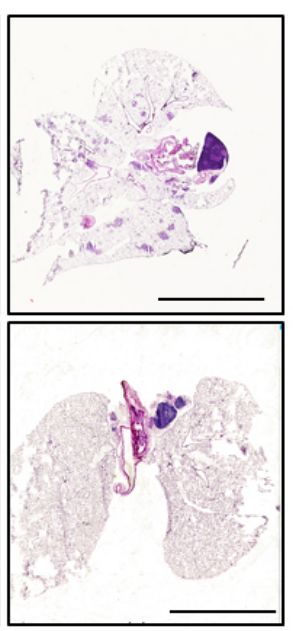

$10 \mathrm{~mm}$

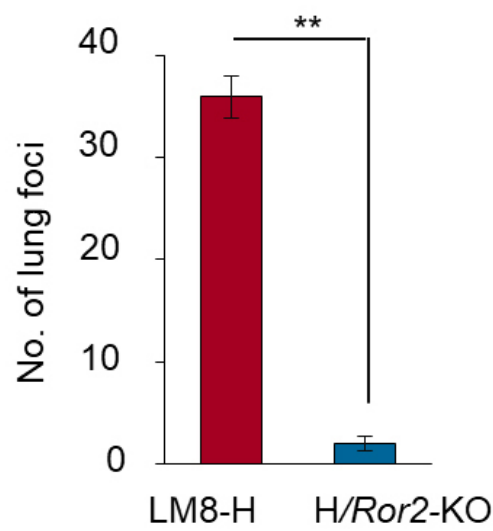

Figure 1. ROR2 regulated lung metastasis of LM8. A: gene set enrichment analysis. Microarray data for LM8-H and LM8-L were analyzed using Kyoto Encyclopedia of Genes and Genomes software to identify significant gene sets. The enrichment plot shows the distribution of genes in the set that are correlated with the Wnt signaling pathway (mm0431); B: mRNA expression levels of Ror2, Ror1, and Fzd1 in LM8-H and LM8-L analyzed by RT-PCR. The numbers below the bands indicate the corresponding expression levels relative to $\beta$-actin; C: ROR2 protein expression levels in LM8-L, LM8-H, and H/Ror2-KO examined by western blotting; D: proliferation rates of LM8-H, LM8-L, and $\mathrm{H} /$ Ror2-KO cultured under adhesion conditions for 3 days; $\mathrm{E}$ : representative lung images stained with hematoxylin-eosin (left) and the number of lung foci bigger than $1 \mathrm{~mm}$ in diameter (right) at day 20 after intravenous injection of LM8-H and H/Ror2-KO. Scale bars: $10 \mathrm{~mm}, n=5,{ }^{\star} P<0.01$. ROR2: receptor tyrosine kinase-like orphan receptor 2; LM8-H: LM8 cell line with high metastatic ability; LM8-L: LM8 cell line with low metastatic ability; Fzd1: Frizzled class receptor 1; H/Ror2-KO: LM8-H knocked out of Ror2; n.s: not significant 
A

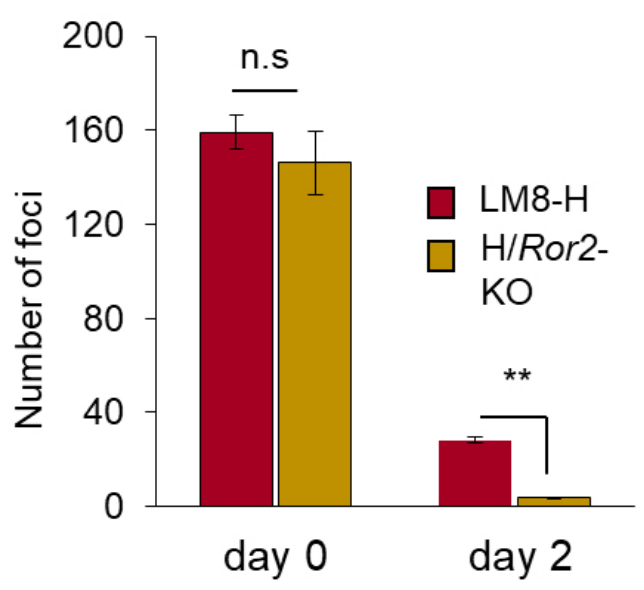

LM8-H $\quad \mathrm{H} /$ Ror2-KO

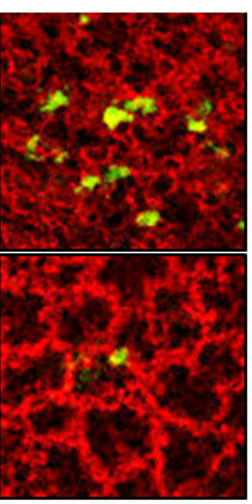

B
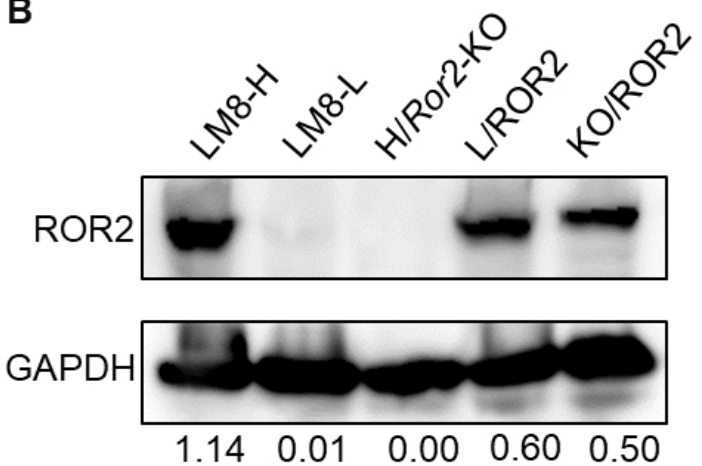

C

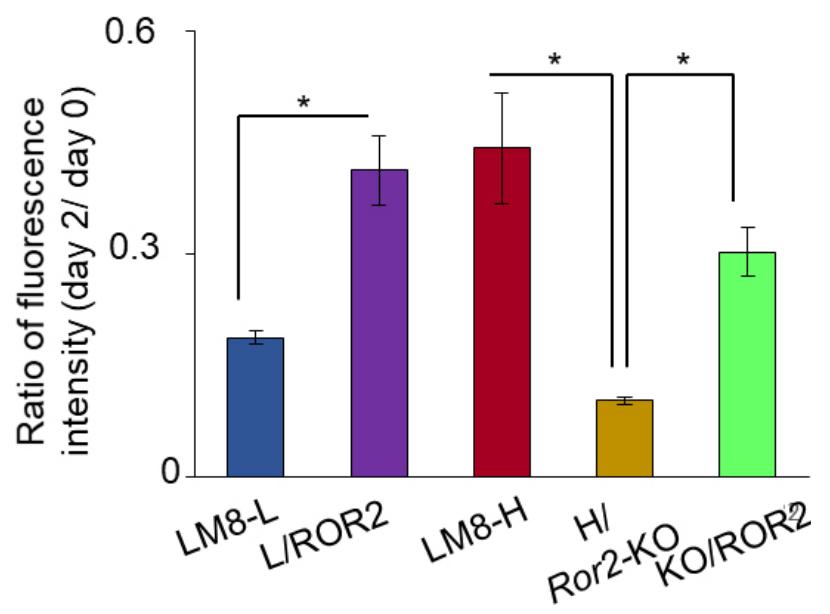

Figure 2. ROR2 regulated the survival of LM8 in lung capillaries. A: fluorescently labeled LM8 cells in the lungs were quantified using an inverted confocal fluorescence microscope (top). Data are shown as the mean \pm SD of three fields of fluorescently labeled cells in each lung on the indicated days. $n=3,{ }^{*} P<0.01$. Representative image of LM8 cells in lungs on the indicated days (bottom). Red: blood vessel, Green: tumor cell, Black: lung tissue. Scale: $50 \mu \mathrm{m}$; B: ROR2 protein level in the indicated LM8 sublines. The numbers below the bands indicate the corresponding ROR2 expression levels relative to GAPDH; C: fluorescence intensity in lung lysates prepared from mice injected with the indicated LM8 sublines was quantified on day 0 (30 min) and day 2 ( $48 \mathrm{~h}$ ). Data are shown as the ratio of day 2 fluorescence intensity normalized to day $0 . n=3$, ${ }^{\star} P<0.05$. ROR2: receptor tyrosine kinase-like orphan receptor 2; LM8-H: LM8 cell line with high metastatic ability; LM8-L: LM8 cell line with low metastatic ability; H/Ror2-KO: LM8-H knocked out of Ror2; L/ROR2: LM8-L expressing ROR2; KO/ROR2: H/Ror2-KO expressing ROR2; n.s: not significant

than $90 \%$ of tumor cells entering the capillaries ${ }^{[22]}$. The LM8 sublines were examined for sensitivity to mechanical stress in vitro using a hypotonic buffer that causes hypotonic cell swelling, an established perturbation method that examines the strength against mechanical stress by inducing elongation of the plasma membrane in a well-controlled manner ${ }^{[23]}$. Incubation of LM8-H and H/Ror2-KO in hypotonic buffer for $30 \mathrm{~min}$ reduced viability by less than half in both sublines compared to incubation in isotonic buffer [Supplementary Figure 3], indicating that ROR2 was not involved in the resistance to mechanical stress in the lung capillaries. The differences in anoikis resistance among the LM8 sublines were examined in terms of viability after culturing for $24 \mathrm{~h}$ on poly-HEMA-coated dishes (under low adhesion conditions) using fluorescent dyes that differentially label live and dead cells. The LM8 sublines with low ROR2 expression (LM8-L and H/Ror2-KO) significantly increased anoikis, and those with high ROR2 expression (L/ROR2 and KO/ROR2) significantly decreased anoikis [Figure 3A]. It has been reported that the activity of Akt was upregulated in anoikis resistant human OS cells $^{[24]}$. Therefore, we investigated the involvement of AKT signaling in LM8 ROR2-induced anoikis resistance. First, AKT activation (Ser473 phospho- 
A

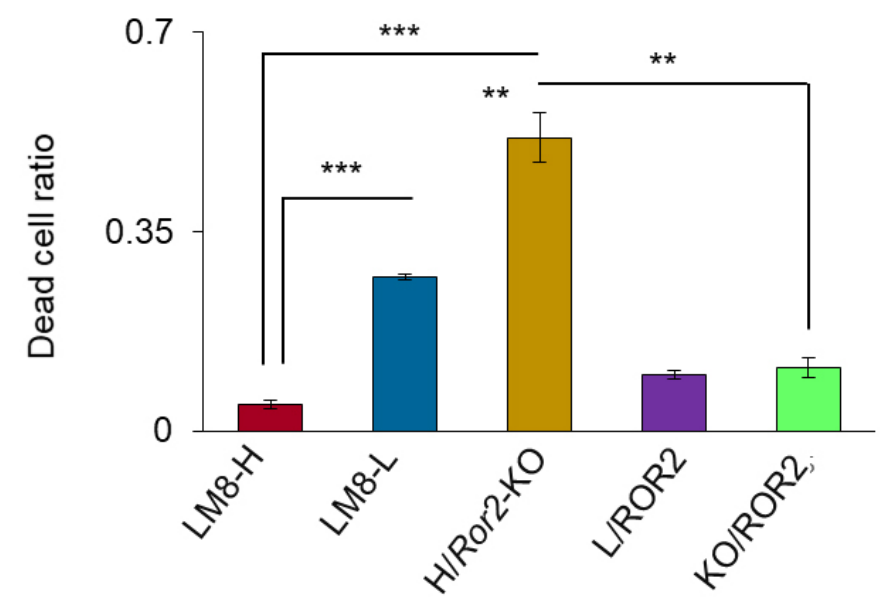

C

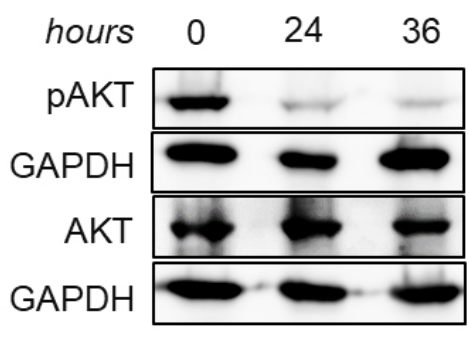

$\begin{array}{llll}\text { pAKT/ } & 0.70 & 0.10 & 0.07\end{array}$

B
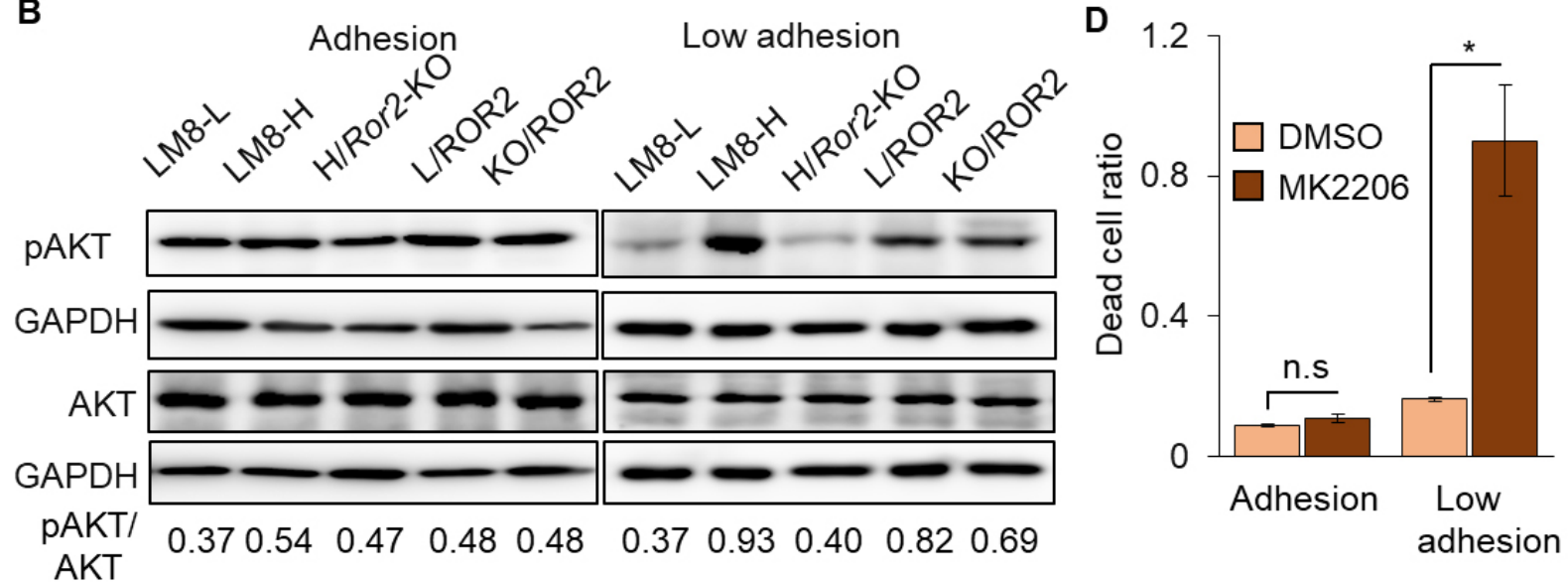

Figure 3. AKT signaling was involved in anoikis regulation by ROR2 in LM8. A: fluorescence intensities of live and dead cells labeled with different dyes were measured using appropriate filters after $24 \mathrm{~h}$ of culture under low adhesion conditions as described in the Methods. The dead cell ratio was calculated by dividing the fluorescence intensity of the dead cells by the total fluorescence intensity. $n=3,{ }^{\star} P<0.05,{ }^{\star \star} P<0.01,{ }^{\star \star \star} P<0.001 ; \mathrm{B}$ : PAKT and AKT levels in LM8 sublines cultured under adhesion (left) and low adhesion (right) conditions analyzed by western blotting. The pAKT/AKT at the bottom of the figure shows the ratio of pAKT to AKT levels normalized by GAPDH levels; C: pAKT and AKT levels in LM8-H treated with MK2206 for indicated time under low adhesion conditions. The western blotting experiments were repeated three times, and representative data are shown; D: dead cell ratio of LM8-H cells cultured with MK2206 or solvent only (DMSO) for $24 \mathrm{~h}$ under adhesion or low adhesion conditions. Dead cell ratios are shown as normalized values for cells treated with MK2206 vs. those for untreated cells. $n=3,{ }^{\star} P<0.05$. ROR2: receptor tyrosine kinase-like orphan receptor 2; LM8-H: LM8 cell line with high metastatic ability; LM8-L: LM8 cell line with low metastatic ability; H/Ror2-KO: LM8-H knocked out of Ror2; n.s: not significant; DMSO: dimethyl sulfoxide; L/ROR2: LM8-L expressing ROR2; KO/ROR2: H/Ror2-KO expressing ROR2

AKT/AKT ratio) was examined in LM8 sublines with different ROR2 expression levels. Under adhesion conditions, AKT activation was not related to ROR2 expression levels. However, higher AKT activation was observed in the LM8 sublines with high ROR2 expression (LM8-H, L/ROR2, and KO/ROR2) compared to the LM8 sublines with low ROR2 expression (LM8-L and H/Ror2-KO) under low adhesion conditions [Figure 3B]. Second, the correlation between AKT activation and anoikis was examined using MK2206, an AKT inhibitor. AKT activation in LM8-H under low adhesion conditions was significantly suppressed by MK2206 [Figure 3C], resulting in a significant increase in anoikis in these cells [Figure 3D]. These results suggest a novel ROR2 function involved in LM8 anoikis resistance through AKT activation.

\section{DISCUSSION}

In the present study, we identified a novel function of ROR2 in lung metastases of the mouse OS cell line LM8; ROR2 may contribute to OS cell survival in lung capillaries by increasing anoikis resistance through 
AKT activation. Previous studies of various tumor cells have reported important functions of ROR2 in migration and invasion ${ }^{[25-29]}$, which are essential metastasis initiation steps in the pre-circulatory processes. The lung metastasis model used in this study was designed to focus on the post-circulatory processes of metastasis, allowing us to discover another important function of ROR2 in the lung metastatic process.

Previous study using the same lung metastasis model revealed that CYGB function is crucial for LM8 extravasation ability, and Cygb was previously identified as a LEF1-regulated gene ${ }^{[16]}$. Although Ror 2 is one of the Wnt signaling-related genes, the ROR2 expression level did not correlate with the LEF1 expression level [Supplementary Figure 4A]. In contrast, Cygb mRNA levels were significantly reduced in LM8-L and H/Ror2-KO [Supplementary Figure $4 \mathrm{~B}$ ], suggesting that ROR2 signaling may be an alternative CYGB regulator independent of LEF-1 signaling in LM8. In fact, overexpression of ROR2 in LM8-L resulted in significantly increased endothelial transmigration abilities while knocking out of Ror2 in LM8-H resulted in significantly decreased endothelial transmigration abilities [Supplementary Figure $4 \mathrm{C}$ ]. These results are similar to those observed in the previous study using CYGB-overexpressing $\mathrm{LM} 8-\mathrm{L}$ and $\mathrm{Cyg}$ - $\mathrm{KO}$ LM8- $\mathrm{H}^{[16]}$. Together with our previous study of the LEF1-CYGB axis, this study of ROR2-CYGB axis provided new insight into the mechanism of Wnt-signaling in OS lung metastasis. Although the Wnt5a-ROR2 axis has been reported to induce the migration of human OS cells $^{[25,28,30,31]}$, we found that recombinant Wnt5a did not promote transmigration of LM8-H [Supplementary Figure 5], suggesting that Wnt5a-ROR2 signaling was not involved in the post-circulatory process of lung metastasis in LM8 sublines.

We demonstrated that the anoikis resistance regulated by ROR2 was dependent on AKT activation and that AKT inhibition reduced ROR2-induced anoikis resistance. Since Ror2 expression level did not affect LM8 cell proliferation under adhesion conditions [Figure 1D], the increased viability of LM8 sublines with high ROR2 expression is not due to increased proliferation. The AKT inhibitor used here, MK2206, is currently in phase II clinical trials for recurrent and advanced endometrial cancer. In addition to inhibiting tumor growth in neuroblastoma and colorectal cancer $^{[32]}$, MK2206 also impairs the proliferation of human OS cells such as $\mathrm{U}_{2} \mathrm{OS}$ and $\mathrm{HOS}^{[33]}$. MK2206 was found to reduce LM8-H viability only under low adhesion conditions, and no cell death was observed with MK2206 treatment under adhesion conditions. These results indicate that MK2206 might be an ideal drug to specifically kill OS cells that have lost anchorage dependency, such as cells that have acquired the ability to invade and metastasize.

In conclusion, our findings suggested that the ROR2-AKT axis enhanced OS lung metastasis through anoikis resistance and that MK2206 enhances the induction of apoptosis in OS cells under low adhesion conditions. These discoveries may shed light on specific stages of the metastatic process and provide clues for the development of effective OS treatment.

\section{DECLARATIONS}

\section{Acknowledgments}

We thank Sakiko Yonezawa and Ngoc Thi Hong Hoang for technical advice of Western blotting, qPCR and RT-PCR. We also thank the Open Research Facilities for Life Science and Technology, Tokyo Institute of Technology for technical assistance. We would like to thank Editage (www.editage.com) for English language editing.

\section{Authors' contributions}

Designed overall projects: Tran DTP, Kuchimaru T, Kizaka-Kondoh S

Performed experiments: Tran DTP, Kuchimaru T

Analyzed and interpreted data: Tran DTP, Kuchimaru T, Pongsuchart M, Nguyen TK, Co Soriano JC, Kadonosono T, Kizaka-Kondoh S

Wrote the manuscript: Tran DTP, Kizaka-Kondoh S 


\section{Availability of data and materials}

The data that support the findings of this study are available from the corresponding author upon request.

\section{Financial support and sponsorship}

This work was supported by a Grant-in-Aid for Scientific Research on Innovative Areas "Integrative Research on Cancer Microenvironment Networks from the Ministry of Education", Culture, Sports, Science and Technology of Japan" (Kizaka-Kondoh S), and Grant-in-Aid for Young Scientist (B) (Kuchimaru T).

\section{Conflicts of interest}

All authors declared that there are no conflicts of interest.

\section{Ethical approval and consent to participate}

Male $\mathrm{C} 3 \mathrm{H}$ mice were obtained from the Charles River Laboratory, Japan (Yokohama, Japan). All mice used were 6-8 weeks of age and were housed in the animal facilities at the Tokyo Institute of Technology. Animal experiments were performed with the approval of the Animal Ethics Committees of the Tokyo Institute of Technology (No. D20170004-2) and in accordance with the Ethical Guidelines for Animal Experimentation of the Tokyo Institute of Technology.

\section{Consent for publication}

Not applicable.

\section{Copyright}

(c) The Author(s) 2020.

\section{REFERENCES}

1. de Azevedo JWV, Fernandes TAADM, Fernandes JV, de Azevedo JCV, Lanza DCF, Bezerra CM. Biology and pathogenesis of human osteosarcoma. Oncology Letters 2020;19:1099-1116.

2. Czarnecka AM, Synoradzki K, Firlej W, Bartnik E, Sobczuk P, Fiedorowicz M. Molecular biology of osteosarcoma. Cancers 2020;12:2130.

3. Wen XL, Wu YL, Awadasseid A, Tanaka Y, Zhang W. New advances in canonical Wnt/ $\beta$-catenin signaling in cancer. Cancer Manag Res 2020;12:6987-98.

4. Singla A, Wang J, Yang R, Geller DS, Loeb DM, Hoang BH. Wnt signaling in osteosarcoma. In: Kleinerman E, Gorlick R, editors. Current advances in the science of osteosarcoma. Advances in experimental medicine and biology. New York: Springer, Cham; 2020. pp. 125-39.

5. Zhang AL, He SH, Sun XL, Ding LH, Bao XH, Wang N. Wnt5a promotes migration of human osteosarcoma cells by triggering a phosphatidylinositol-3 kinase/Akt signals. Cancer Cell Int 2014;14:15.

6. Van Amerongen R. Alternative Wnt pathways and receptors. Cold Spring Harb Perspect Biol 2012;4:a007914.

7. Debebe Z, Rathmell WK. Ror2 as a therapeutic target in cancer. Pharmacol Ther 2015;150:143-8.

8. Masiakowski P, Carroll R D. A novel family of cell surface receptors with tyrosine kinase-like domain. J Biol Chem 1992;267:26181-90.

9. Oishi I, Takeuchi S, Hashimoto R, et al. Spatio-temporally regulated expression of receptor tyrosine kinases, mRor1, mRor2, during mouse development: implications in development and function of the nervous system. Genes Cells 1999;4:41-56.

10. Oishi I, Suzuki H, Onishi N, et al. The receptor tyrosine kinase Ror2 is involved in non-canonical Wnt5a/JNK signalling pathway. Genes Cells 2003;8:645-54.

11. Nishita M, Yoo SK, Nomachi A, et al. Filopodia formation mediated by receptor tyrosine kinase Ror2 is required for Wnt5a-induced cell migration. J Cell Biol 2006;175:555-62.

12. Nomachi A, Nishita M, Inaba D, Enomoto M, Hamasaki M, Minami Y. Receptor tyrosine kinase Ror2 mediates Wnt5a-induced polarized cell migration by activating c-Jun N-terminal kinase via actin-binding protein filamin A. J Biol Chem 2008;283:27973-81.

13. Lu BJ, Wang YQ, Wei XJ, et al. Expression of WNT-5a and ROR2 correlates with disease severity in osteosarcoma. Mol Med Rep 2012;5:1033-6.

14. Frisch SM, Screaton RA. Anoikis mechanisms. Curr Opin Cell Biol 2001;13:555-62.

15. Gassmann P, Haier J. The tumor cell-host organ interface in the early onset of metastatic organ colonisation. Clin Exp Metastasis 2008;25:171-81.

16. Pongsuchart M, Kuchimaru T, Yonezawa S, et al. Novel lymphoid enhancer-binding factor 1-cytoglobin axis promotes extravasation of osteosarcoma cells into the lungs. Cancer Sci 2018;109:2746-56. 
17. Mashiko D, Young SAM, Muto M, et al. Feasibility for a large scale mouse mutagenesis by injecting CRISPR/Cas plasmid into zygotes. Dev Growth Differ 2014;56:122-9.

18. Abràmoff MD, Magalhães PJ, Ram SJ. Image processing with imagej second edition. Biophotonics. 2004;11:36-42

19. Subramanian A, Tamayo P, Mootha VK, et al. Gene set enrichment analysis: a knowledge-based approach for interpreting genome-wide expression profiles. Proc Natl Acad Sci U S A 2005;102:15545-50.

20. Kanehisa M. KEGG: Kyoto encyclopedia of genes and genomes. Nucleic Acids Res 2000;28:27-30.

21. Zeidman I. The fate of circulating tumors cells. I. Passage of cells through capillaries. Cancer Res 1961;21:38-9.

22. Weiss L, Harlos JP, Elkin G. Mechanism of mechanical trauma to ehrlich ascites tumor cells in vitro and its relationship to rapid intravascular death during metastasis. Int J Cancer 1989;44:143-8.

23. Furlow PW, Zhang S, Soong TD, et al. Mechanosensitive pannexin-1 channels mediate microvascular metastatic cell survival. Nat Cell Biol 2015;17:943-52.

24. Díaz-Montero CM, Wygant JN, McIntyre BW. PI3-K/Akt-mediated anoikis resistance of human osteosarcoma cells requires Src activation. Eur J Cancer 2006;42:1491-500.

25. Enomoto M, Hayakawa S, Itsukushima S, et al. Autonomous regulation of osteosarcoma cell invasiveness by Wnt5a/Ror2 signaling. Oncogene 2009;28:3197-208.

26. O'Connell MP, Fiori JL, Xu M, et al. The orphan tyrosine kinase receptor, ROR2, mediates Wnt5A signaling in metastatic melanoma. Oncogene 2010;29:34-44.

27. Leong HS, Robertson AE, Stoletov K, et al. Invadopodia are required for cancer cell extravasation and are a therapeutic target for metastasis. Cell Rep 2014;8:1558-70.

28. Nomachi A, Nishita M, Inaba D, Enomoto M, Hamasaki M, Minami Y. Receptor tyrosine kinase Ror2 mediates Wnt5a-induced polarized cell migration by activating c-Jun N-terminal kinase via actin-binding protein Filamin A. J Biol Chem 2008;283:27973-81.

29. Henry CE, Llamosas E, Djordjevic A, Hacker NF, Ford CE. Migration and invasion is inhibited by silencing ROR1 and ROR2 in chemoresistant ovarian cancer. Oncogenesis 2016;5:e226.

30. Nishita M, Park SY, Nishio T, et al. Ror2 signaling regulates Golgi structure and transport through IFT20 for tumor invasiveness. Sci Rep 2017;7:1-15.

31. Dai B, Yan T, Zhang AL. ROR2 receptor promotes the migration of osteosarcoma cells in response to Wnt5a. Cancer Cell Int 2017;17:1-9.

32. Li ZJ, Ramalingam S, Yan S, Thiele CJ. Abstract 4342: the allosteric Akt inhibitor MK-2206 inhibits neuroblastoma tumor cell growth in vitro and in vivo. Cancer Res 2011;71:4342.

33. Hofmann C, Obermeier F, Artinger M, et al. Cell-cell contacts prevent anoikis in primary human colonic epithelial cells. Gastroenterology 2007;132:587-600. 